\title{
Cycles of even lengths modulo $k$
}

\author{
Ajit A. Diwan \\ Department of Computer Science and Engineering, \\ Indian Institute of Technology Bombay, \\ Mumbai, 400076, India.
}

January 19, 2009

\begin{abstract}
Thomassen [9] conjectured that for all natural numbers $k>0$ and $m$, every graph of minimum degree $k+1$ contains a cycle of length congruent to $2 m$ modulo $k$. We prove that this is true for $k \geq 2$ if the minimum degree is $2 k-1$, which improves the previously known bound of $3 k-2$. We also show that Thomassen's conjecture is true for $m=2$.
\end{abstract}

Keywords: cycle lengths; minimum degree

\section{Introduction}

The study of cycle lengths in a graph modulo a positive integer $k$ was initiated by Erdős and Burr [6], who conjectured that for every odd positive integer $k$, there exists a constant $c_{k}$ such that for all natural numbers $m$, every graph of average degree at least $c_{k}$ contains a cycle of length congruent to $m$ modulo $k$. They proved this for $m=2$ and the full conjecture was proved by Bollobás [1], who showed that $c_{k}=2\left((k+1)^{k}-1\right) / k$ suffices. Thomassen $[9,10]$ showed a more general result, that an average degree of $4 k(k+1)$ implies that the graph contains a cycle of length congruent to $2 m$ modulo $k$ for all natural numbers $k>0$ and $m$. He also conjectured that a minimum degree of $k+1$ suffices.

Verstraëte [11] showed that if $k \geq 2$ and the average degree is at least $8 k$, then there exist $k$ cycles of consecutive even lengths, which implies that for all natural numbers $m$, there exists a cycle of length congruent to $2 m$ modulo $k$. Fan [7] showed that if $k \geq 2$ and the minimum degree is $3 k-2$, then there exist $k$ cycles 
with consecutive even lengths, or consecutive odd lengths. This implies that for odd $k \geq 2$, if the minimum degree is $3 k-2$, then the graph contains a cycle of length congruent to $2 m$ modulo $k$ for all $m$. We give a simple proof that for $k \geq 2$, a minimum degree of $2 k-1$ suffices to ensure that the graph contains a cycle of length congruent to $2 m$ modulo $k$ for all $m$. The proof is an application of a technique used by Mader in [8].

Thomassen's conjecture is known to be true for $k \leq 4,[3,4,5]$ and for claw-free graphs [2]. For $m=1$, it follows easily by considering a longest path and the edges incident with an endpoint of the path. We prove that it is true for $m=2$.

All graphs considered are simple and finite. We denote the vertex set of a graph $G$ by $V(G)$ and the edge set by $E(G)$. The set of neighbors of a vertex $v$ in a graph $G$ is denoted by $N_{G}(v)$ or simply $N(v)$ when $G$ is understood from the context. If $V \subset V(G)$ then $G-V$ is the subgraph of $G$ obtained by deleting all vertices in $V$ and edges incident with them. The graph $G-(V(G) \backslash V)$ is the subgraph of $G$ induced by $V$.

A path with endpoints $\{u, v\}$ is called a $u-v$ path, and a $u-u$ path is the trivial path containing only the vertex $u$. The length of a path or cycle $P$ is denoted by $l(P)$. A set of paths (or cycles) $\left\{P_{1}, P_{2}, \ldots, P_{r}\right\}$ is said to have distinct lengths modulo $k$ if $l\left(P_{i}\right) \not \equiv l\left(P_{j}\right) \bmod k$ for all $1 \leq i<j \leq r$. A graph $G$ is said to contain all cycles of even lengths modulo $k$, if for all natural numbers $m, G$ contains a cycle of length congruent to $2 m$ modulo $k$. If $P$ and $Q$ are graphs, we denote by $P \cup Q$ the graph with vertex set $V(P) \cup V(Q)$ and edge set $E(P) \cup E(Q)$. To simplify the notation, we will consider an edge to be a graph with two vertices, where necessary.

\section{Main Result}

Theorem 1 Let $k \geq 2$ be an integer and $G$ a graph with minimum degree $2 k-1$. For all natural numbers $m, G$ contains a cycle of length congruent to $2 m$ modulo $k$.

We will assume throughout that $k \geq 2$ is a fixed integer. We will be considering ordered pairs of the form $(G, K)$, where $K$ is a proper complete subgraph of $G$, possibly empty.

Definition 1 The ordered pair $(G, K)$ contains a configuration of type $\boldsymbol{A}$ if $V(G) \backslash$ $V(K)$ contains a vertex $u$ such that $|N(u) \cap V(K)| \geq 2 k-1$.

Definition 2 The ordered pair $(G, K)$ contains a configuration of type $\boldsymbol{B}$ if $V(G) \backslash$ $V(K)$ contains two vertices $u, v$ such that $|N(u) \cap V(K)| \geq 2 k-2,|N(v) \cap V(K)| \geq$ $2 k-2$, and there exists a $u-v$ path $P$ in $G-V(K)$. 
Definition 3 The ordered pair $(G, K)$ contains a configuration of type $\boldsymbol{C}$ and rank $r$, if $V(G) \backslash V(K)$ contains two vertices $u, v$ such that $|N(u) \cap V(K)| \geq 2(k-r)+1$, $|N(v) \cap V(K)| \geq 2(k-r)+1$, and there exists a set $\left\{P_{1}, P_{2}, \ldots, P_{r}\right\}$ of $r u-v$ paths in $G-V(K)$ having distinct lengths modulo $k$.

Definition 4 The ordered pair $(G, K)$ contains a configuration of type $\boldsymbol{D}$ and rank $r$, if $V(G) \backslash V(K)$ contains three vertices $u, w, v$ satisfying the following properties.

1. uw and wv are edges in $G-V(K)$.

2. There exist vertices $\left\{u^{\prime}, w^{\prime}, v^{\prime}\right\} \subseteq V(G) \backslash V(K)$ and vertex-disjoint paths $P_{u}$, $P_{w}, P_{v}$ in $G-V(K)$ such that $\left|N\left(u^{\prime}\right) \cap V(K)\right| \geq 2(k-r)-1,\left|N\left(w^{\prime}\right) \cap V(K)\right| \geq$ $2(k-r),\left|N\left(v^{\prime}\right) \cap V(K)\right| \geq 2(k-r)$, and $P_{z}$ is a $z-z^{\prime}$ path for all $z \in\{u, v, w\}$. Note that the vertex $z^{\prime}$ may be the same as the vertex $z$, for $z \in\{u, v, w\}$.

3. There is a set $\left\{P_{1}, P_{2}, \ldots, P_{r}\right\}$ of $r u-v$ paths in $G-V(K)$ having distinct lengths modulo $k$, such that $P_{i}$ is internally vertex-disjoint from $P_{u}, P_{w}$ and $P_{v}$ for $1 \leq i \leq r$. Also, one of the paths in $\left\{P_{1}, P_{2}, \ldots, P_{r}\right\}$ has length congruent to 0 modulo $k$.

Lemma 1 Let $G$ be a graph and $K$ a proper complete subgraph of $G$. If every vertex in $V(G) \backslash V(K)$ has degree at least $2 k-1$ in $G$, then either $G-V(K)$ contains cycles of all even lengths modulo $k$, or the ordered pair $(G, K)$ contains a configuration of one of the types $\boldsymbol{A}, \boldsymbol{B}, \boldsymbol{C}, \boldsymbol{D}$. If the configuration is of type $\boldsymbol{C}$, then the rank is at least two and at most $k$. If the configuration is of type $\boldsymbol{D}$, then the rank is at least two and less than $k$.

Proof: Let $(G, K)$ be a counterexample that minimizes $|V(G) \backslash V(K)|$ and subject to this condition, minimizes $|V(K)|$. If $|V(G) \backslash V(K)|=1$, the only vertex $u \in$ $V(G) \backslash V(K)$ has degree at least $2 k-1$, hence $|N(u) \cap V(K)| \geq 2 k-1$. This implies $(G, K)$ contains a configuration of type $\mathbf{A}$, a contradiction.

Suppose $|V(G) \backslash V(K)|>1$. We consider two cases.

Case 1.

Suppose there exists a vertex $x \in V(G) \backslash V(K)$ that is adjacent to all vertices in $V(K)$. Let $K^{\prime}$ be the complete subgraph of $G$ induced by $V(K) \cup\{x\}$. Then the ordered pair $\left(G, K^{\prime}\right)$ satisfies the hypothesis of Lemma 1 , and by the minimality of $(G, K)$, either $G-V\left(K^{\prime}\right)$ contains cycles of all even lengths modulo $k$ or $\left(G, K^{\prime}\right)$ contains a configuration of one of the four types. Since $G-V\left(K^{\prime}\right)$ is a subgraph of $G-V(K)$, we may assume that the latter holds. Now we show that in each case, the configuration in $\left(G, K^{\prime}\right)$ can be modified to either find cycles of all even lengths 
modulo $k$ in $G-V(K)$, or get a configuration of one of the four types in $(G, K)$, contradicting the fact that $(G, K)$ is a counterexample.

Case 1.1

Suppose $\left(G, K^{\prime}\right)$ contains a configuration of type $\mathbf{A}$. Let $u$ be a vertex in $V(G) \backslash$ $V\left(K^{\prime}\right)$ such that $\left|N(u) \cap V\left(K^{\prime}\right)\right| \geq 2 k-1$. If $u$ is not adjacent to $x$, then $\mid N(u) \cap$ $V(K) \mid \geq 2 k-1$ and $(G, K)$ contains a configuration of type $\mathbf{A}$. If $u$ is adjacent to $x$, then $|N(u) \cap V(K)| \geq 2 k-2$ and since $x$ is adjacent to every vertex in $V(K)$, $|N(x) \cap V(K)|=|V(K)| \geq 2 k-2$. The edge $u x$ implies that $(G, K)$ contains a configuration of type $\mathbf{B}$.

Case 1.2

Suppose $\left(G, K^{\prime}\right)$ contains a configuration of type B. Let $u, v$ be vertices in $V(G) \backslash$ $V\left(K^{\prime}\right)$ such that $\left|N(u) \cap V\left(K^{\prime}\right)\right| \geq 2 k-2,\left|N(v) \cap V\left(K^{\prime}\right)\right| \geq 2 k-2$ and there is a $u-v$ path $P$ in $G-V\left(K^{\prime}\right)$.

If $x$ is not adjacent to any of the vertices $\{u, v\}$, then $u, v$ satisfy the same properties with $K^{\prime}$ replaced by $K$, and $(G, K)$ contains the same configuration of type $\mathbf{B}$.

If $x$ is adjacent to $u$ but not adjacent to $v$, then $|N(v) \cap V(K)| \geq 2 k-2$ and hence $|N(x) \cap V(K)| \geq 2 k-2$. Also, $P \cup x u$ is an $x-v$ path in $G-V(K)$. Hence $(G, K)$ contains a configuration of type B. A symmetrical argument holds if $x$ is adjacent to $v$ but not adjacent to $u$.

Suppose $x$ is adjacent to both $u$ and $v$. Then $|N(u) \cap V(K)| \geq 2 k-3, \mid N(v) \cap$ $V(K) \mid \geq 2 k-3$ and hence $|N(x) \cap V(K)| \geq 2 k-3$. If $l(P) \equiv 0 \bmod k$ and $k>2$, then $P$ and $Q=u x \cup x v$ are two $u-v$ paths in $G-V(K)$ of distinct lengths modulo $k$, hence $(G, K)$ contains a configuration of type $\mathbf{C}$ and rank two. If $l(P) \equiv 0 \bmod k$ and $k=2$, then $P \cup Q$ is an even cycle in $G-V(K)$, hence $G-V(K)$ contains cycles of all even lengths modulo 2. If $l(P) \not \equiv 0 \bmod k$, then the edge $u x$ and the path $P \cup v x$ are two $u-x$ paths in $G-V(K)$ of distinct lengths modulo $k$, hence $(G, K)$ contains a configuration of type $\mathbf{C}$ and rank two.

Case 1.3

Suppose $\left(G, K^{\prime}\right)$ contains a configuration of type $\mathbf{C}$ and rank $r$, for some $2 \leq$ $r \leq k$. Let $u, v$ be vertices in $V(G) \backslash V\left(K^{\prime}\right)$ such that $\left|N(u) \cap V\left(K^{\prime}\right)\right| \geq 2(k-r)+1$, $\left|N(v) \cap V\left(K^{\prime}\right)\right| \geq 2(k-r)+1$ and let $\left\{P_{1}, P_{2}, \ldots, P_{r}\right\}$ be the set of $r u-v$ paths in $G-V\left(K^{\prime}\right)$ having distinct lengths modulo $k$.

If $x$ is not adjacent to any of the vertices $\{u, v\}$, then $(G, K)$ also contains the same configuration of type $\mathbf{C}$ and rank $r$.

If $x$ is adjacent to $u$ but not to $v$, then $|N(v) \cap V(K)| \geq 2(k-r)+1$ and hence $|N(x) \cap V(K)| \geq 2(k-r)+1$. The paths $P_{i} \cup x u$ for $1 \leq i \leq r$ are $x-v$ paths in 
$G-V(K)$ having distinct lengths modulo $k$. Hence $(G, K)$ contains a configuration of type $\mathbf{C}$ and rank $r$. A symmetrical argument holds if $x$ is adjacent to $v$ but not adjacent to $u$.

Suppose $x$ is adjacent to both $u$ and $v$. If $r=k$, then the cycles $u x \cup x v \cup P_{i}$ for $1 \leq i \leq k$ have distinct lengths modulo $k$, hence $G-V(K)$ contains cycles of all even lengths modulo $k$. If $r<k$, then $|N(u) \cap V(K)| \geq 2(k-r),|N(v) \cap V(K)| \geq 2(k-r)$ and hence $|N(x) \cap V(K)| \geq 2(k-r)$. If none of the paths $P_{1}, P_{2}, \ldots, P_{r}$ has length congruent to 0 modulo $k$, then the edge $u x$ and the paths $P_{i} \cup v x$ for $1 \leq i \leq r$ are $r+1 u-x$ paths in $G-V(K)$ with distinct lengths modulo $k$, and $(G, K)$ contains a configuration of type $\mathbf{C}$ and rank $r+1$.

Suppose one of the paths $P_{1}, P_{2}, \ldots, P_{r}$ has length congruent to 0 modulo $k$. Then by relabeling the vertex $x$ as $w$, choosing the vertices $u^{\prime}, w^{\prime}, v^{\prime}$ to be the vertices $u, w, v$ respectively, and the paths $P_{u}, P_{w}, P_{v}$ to be trivial, we get a configuration of type $\mathbf{D}$ and rank $r$ in $(G, K)$.

Case 1.4

Finally, suppose $\left(G, K^{\prime}\right)$ contains a configuration of type $\mathbf{D}$ and rank $r$, for some $2 \leq r<k$. Let $u, w, v$ be the three vertices in $V(G) \backslash V\left(K^{\prime}\right)$ such that $u w, w v$ are edges in $G$. Let $u^{\prime}, w^{\prime}, v^{\prime}$ be the vertices in $V(G) \backslash V\left(K^{\prime}\right)$ such that $\mid N\left(u^{\prime}\right) \cap$ $V\left(K^{\prime}\right)|\geq 2(k-r)-1| N,\left(w^{\prime}\right) \cap V\left(K^{\prime}\right) \mid \geq 2(k-r)$ and $\left|N\left(v^{\prime}\right) \cap V\left(K^{\prime}\right)\right| \geq 2(k-r)$ and let $P_{u}, P_{w}, P_{v}$ be the vertex-disjoint $u-u^{\prime}, w-w^{\prime}$ and $v-v^{\prime}$ paths in $G-V\left(K^{\prime}\right)$, respectively. Let $\left\{P_{1}, P_{2}, \ldots, P_{r}\right\}$ be the set of $r u-v$ paths in $G-V\left(K^{\prime}\right)$ that are internally vertex-disjoint from the paths $P_{u}, P_{w}, P_{v}$ and have distinct lengths modulo $k$.

If $x$ is not adjacent to any of the vertices $\left\{u^{\prime}, w^{\prime}, v^{\prime}\right\}$, it is clear that $(G, K)$ contains the same configuration of type $\mathbf{D}$ and rank $r$. If $x$ is adjacent to exactly one of the vertices $\left\{u^{\prime}, w^{\prime}, v^{\prime}\right\}$, then $|N(x) \cap V(K)| \geq 2(k-r)$. If $x$ is adjacent only to $z^{\prime}$ for some $z \in\{u, w, v\}$, replace the vertex $z^{\prime}$ by the vertex $x$ and the path $P_{z}$ by the path $P_{z} \cup x z^{\prime}$. This gives a configuration of type $\mathbf{D}$ and rank $r$ in $(G, K)$.

Suppose $x$ is adjacent to $u^{\prime}$ and $v^{\prime}$ but not to $w^{\prime}$. Then $\left|N\left(w^{\prime}\right) \cap V(K)\right| \geq 2(k-r)$, $\left|N\left(v^{\prime}\right) \cap V(K)\right| \geq 2(k-r)-1$ and hence $|N(x) \cap V(K)| \geq 2(k-r)$. Replace the vertex $u^{\prime}$ by $x$ and the path $P_{u}$ by the path $P_{u} \cup x u^{\prime}$. Now interchanging the labels of the vertices $\{u, v\}$, labeling $x$ as $v^{\prime}$ and $v^{\prime}$ as $u^{\prime}$, gives a configuration of type $\mathbf{D}$ and rank $r$ in $(G, K)$.

Suppose $x$ is adjacent to $w^{\prime}$ and $u^{\prime}$ and may or may not be adjacent to $v^{\prime}$. Then $\left|N\left(w^{\prime}\right) \cap V(K)\right| \geq 2(k-r)-1,\left|N\left(v^{\prime}\right) \cap V(K)\right| \geq 2(k-r)-1$ and hence $|N(x) \cap V(K)| \geq 2(k-r)-1$. Let $Q_{i}=x u^{\prime} \cup P_{u} \cup P_{i} \cup P_{v}$ and $Q_{i}^{\prime}=x w^{\prime} \cup$ $P_{w} \cup w u \cup P_{i} \cup P_{v}$ for $1 \leq i \leq r$, be $2 r x-v^{\prime}$ paths in $G-V(K)$. If amongst 
the $2 r$ paths $\left\{Q_{1}, Q_{1}^{\prime}, \ldots, Q_{r}, Q_{r}^{\prime}\right\}$, there are $r+1$ paths of distinct lengths modulo $k$, then $(G, K)$ contains a configuration of type $\mathbf{C}$ and rank $r+1$, with $x$ and $v^{\prime}$ as the two required vertices. Similarly, let $S_{i}=w^{\prime} x \cup x u^{\prime} \cup P_{u} \cup P_{i} \cup P_{v}$ and $S_{i}^{\prime}=P_{w} \cup w u \cup P_{i} \cup P_{v}$ for $1 \leq i \leq r$, be $2 r w^{\prime}-v^{\prime}$ paths in $G-V(K)$. If amongst the $2 r$ paths $\left\{S_{1}, S_{1}^{\prime}, \ldots, S_{r}, S_{r}^{\prime}\right\}$ there are $r+1$ paths of distinct lengths modulo $k$, then $(G, K)$ contains a configuration of type $\mathbf{C}$ and rank $r+1$, with $w^{\prime}$ and $v^{\prime}$ as the required vertices.

Suppose both sets of paths $\left\{Q_{1}, Q_{1}^{\prime}, \ldots, Q_{r}, Q_{r}^{\prime}\right\}$ and $\left\{S_{1}, S_{1}^{\prime}, \ldots, S_{r}, S_{r}^{\prime}\right\}$ contain at most $r$ paths of distinct lengths modulo $k$. Note that $l\left(Q_{i}\right) \equiv l\left(P_{i}\right)+C_{1} \bmod k$ for some constant $C_{1}$ and all $1 \leq i \leq r$, which implies that $\left\{Q_{1}, \ldots, Q_{r}\right\}$ have distinct lengths modulo $k$. Similarly, $l\left(Q_{i}^{\prime}\right) \equiv l\left(P_{i}\right)+C_{2} \bmod k$ for some constant $C_{2}$. Also $l\left(S_{i}\right)=l\left(Q_{i}\right)+1$ and $l\left(S_{i}^{\prime}\right)=l\left(Q_{i}^{\prime}\right)-1$. Suppose $l\left(P_{i}\right) \equiv a \bmod k$ for some $1 \leq i \leq r$ and natural number $a$. Then $l\left(Q_{i}^{\prime}\right) \equiv a+C_{2} \bmod k$ and there exists an index $j$ such that $l\left(Q_{j}\right) \equiv a+C_{2} \bmod k$. Therefore $l\left(S_{j}\right) \equiv a+C_{2}+1 \bmod k$. Hence, there is an index $m$ such that $l\left(S_{m}^{\prime}\right) \equiv a+C_{2}+1 \bmod k$, which implies $l\left(P_{m}\right) \equiv a+2 \bmod k$. Since this holds for all paths $P_{i}$, and there exists a path of length congruent to 0 modulo $k$, there must be paths of all even lengths modulo $k$ in $\left\{P_{1}, P_{2}, \ldots, P_{r}\right\}$. Then $u w \cup w v \cup P_{i}$ for $1 \leq i \leq r$ are cycles of all even lengths modulo $k$ in $G-V(K)$. Note that this case can occur only when $k$ is even and $r=k / 2$.

If $x$ is adjacent to $w^{\prime}$ and $v^{\prime}$ but not to $u^{\prime}$, then $\left|N\left(w^{\prime}\right) \cap V(K)\right| \geq 2(k-r)-1$, $\left|N\left(u^{\prime}\right) \cap V(K)\right| \geq 2(k-r)-1$ and hence $|N(x) \cap V(K)| \geq 2(k-r)-1$. Now, we can use the same argument as before, by interchanging the vertices $u, v$ and $u^{\prime}, v^{\prime}$. Case 2.

Suppose there is no vertex $x \in V(G) \backslash V(K)$ that is adjacent to all vertices in $V(K)$. Let $v$ be any vertex in $V(K)$. For every vertex $u \in N_{G}(v) \backslash V(K)$, let $f(u)$ denote any vertex in $V(K)$ that is not adjacent to $u$. Let $G^{\prime}$ be the graph obtained from $G-\{v\}$ by adding edges $u f(u)$ for all vertices $u \in N_{G}(v) \backslash V(K)$. Let $K^{\prime}=K-\{v\}$.

Now, $\left|V\left(G^{\prime}\right) \backslash V\left(K^{\prime}\right)\right|=|V(G) \backslash V(K)|$ but $\left|V\left(K^{\prime}\right)\right|<|V(K)|$, and every vertex in $V\left(G^{\prime}\right) \backslash V\left(K^{\prime}\right)$ has degree at least $2 k-1$ in $G^{\prime}$. Hence by the minimality of $(G, K)$, either $G^{\prime}-V\left(K^{\prime}\right)$ contains cycles of all even lengths modulo $k$, or $\left(G^{\prime}, K^{\prime}\right)$ contains one of the four types of configurations. Since $G^{\prime}-V\left(K^{\prime}\right)=G-V(K)$ and $\left|N_{G^{\prime}}(u) \cap V\left(K^{\prime}\right)\right|=\left|N_{G}(u) \cap V(K)\right|$ for every vertex $u \in V(G) \backslash V(K)$, it follows that either $G-V(K)$ contains cycles of all even lengths modulo $k$, or $(G, K)$ contains the same configuration as $\left(G^{\prime}, K^{\prime}\right)$.

The proof of Theorem 1 follows immediately from Lemma 1. If $G$ is a graph with minimum degree $2 k-1$, then the ordered pair $(G, \emptyset)$ satisfies the hypothesis of 
Lemma 1 and hence either $G$ contains cycles of all even lengths modulo $k$, or $(G, \emptyset)$ contains a configuration of one of the four types. However, since $K$ is empty, the latter is not possible, and the theorem follows.

Note that the proof shows that the cycles can be chosen such that two adjacent edges are included in all cycles.

\section{Remarks}

As mentioned in the introduction, it is straightforward to verify Thomassen's conjecture for $m=1$. However, we do not know any other natural numbers $m$ for which it is known to be true. We show that it is true for $m=2$.

Theorem 2 Every graph with minimum degree $k+1$ contains a cycle of length congruent to 4 modulo $k$, for all integers $k \geq 2$.

We use the same technique as in the previous section. Here, we need only the configurations of type $\mathbf{A}$ and $\mathbf{C}$, with some modifications. In a configuration of type $\mathbf{A}$ we require $|N(u) \cap V(K)| \geq k+1$. In a configuration of type $\mathbf{C}$ and rank $r$, we require $|N(u) \cap V(K)| \geq k-r+1$ and $|N(v) \cap V(K)| \geq k-r+1$, where the rank $r$ is at least one and at most $k$. The only difference in the proof is that when considering a configuration of type $\mathbf{C}$ and rank $r$, in the case when $x$ is adjacent to both $u$ and $v$, if any of the $u-v$ paths in the configuration has length congruent to 2 modulo $k$, then $G-V(K)$ contains a cycle of length congruent to 4 modulo $k$. If no path has length congruent to 2 modulo $k$, then there are $r+1 u-v$ paths in $G-V(K)$ of distinct lengths modulo $k$, which gives a configuration of type $\mathbf{C}$ and rank $r+1$ in $(G, K)$.

\section{References}

[1] B. Bollobás, Cycles modulo k, Bull. London Math. Soc. 9 (1977), 97-98.

[2] X. Cai and W. Shreve, Pancyclicity mod $k$ of claw-free graphs and $K_{1,4}$-free graphs, Discrete Math. 230 (2001), 113-118.

[3] G. T. Chen and A. Saito, Graphs with a cycle of length divisible by three, J. Combin. Theory Ser. B 60 (1994), 277-292.

[4] N. Dean, A. Kaneko, K. Ota and B. Toft, Cycles modulo 3, DIMACS Technical Report 91-32 (1991). 
[5] N. Dean, L. Lesniak and A. Saito, Cycles of length 0 modulo 4 in graphs, Discrete Math. 121 (1993), 37-49.

[6] P. Erdős, Some recent problems and results in graph theory, combinatorics, and number theory, Proc. Seventh S-E Conf. Combinatorics, Graph Theory and Computing, Utilitas Math., Winnipeg, (1976) 3-14.

[7] G. Fan, Distribution of cycle lengths in graphs, J. Combin. Theory Ser B 84 (2002), 187-202.

[8] W. Mader, Existenz gewisser Konfigurationen in n-gesättigten Graphen und in Graphen genügend großer Kantendichte, Math. Ann. 194 (1971), 295-312.

[9] C. Thomassen, Graph decomposition with applications to subdivisions and path systems modulo $k$, J. Graph Theory 7 (1983), 261-271.

[10] C. Thomassen, 'Paths, Circuits and Subdivisions', Selected Topics in Graph Theory 3 L.W. Beineke and R.J. Wilson, (Editors), Academic Press, New York (1988), pp. 97-132.

[11] J. Verstraëte, On arithmetic progressions of cycle lengths in graphs, Combin. Probab. and Comput., 9 (2000), 369-373. 